\title{
Evaluación de programas de lengua: perspectivas profesionales e investigadoras
}

\author{
JULIO CILLER \\ The University of Texas Rio Grande Valley \\ julio.ciller@utrgv.edu
}

\author{
RAMSÉS ORTÍN \\ The University of Texas Rio Grande Valley \\ ramses.ortin@utrgv.edu
}

\section{Resumen}

Este artículo aboga por el uso de procedimientos evaluativos a nivel de programa para asegurar la calidad y el valor de programas de lengua. Tras revisar el estado general del campo de evaluación de programas de lengua a nivel académico, argumentamos que los beneficios de prácticas evaluativas constantes ayudan a estrechar la relación entre la oferta formativa de un programa y las necesidades de las partes involucradas (estudiantes, profesores, comunidad). Proponemos el uso de los estándares de CEA como guía para realizar una evaluación completa de programas de lengua a través de herramientas diseñadas específicamente para las características particulares de cada programa. Finalmente, se pretende fomentar el desarrollo y la publicación de estudios de caso, recursos, y/o revistas para realzar el estatus de la evaluación de programas en el campo de la lingüística aplicada.

\section{Palabras clave}

Evaluación de programas; enseñanza de idiomas; estándares educativos; calidad educativa. 


\title{
Language Program Evaluation: Professional and Research Perspectives
}

\author{
Abstract \\ The purpose of this article is to advocate for the use of ongoing evaluative processes at the \\ programmatic level in order to ensure language program quality and worth. After a thorough \\ review of the field of language program evaluation, we argue that the benefits of evaluation \\ cannot but strengthen the relationship between an academic program and stakeholders' needs \\ (students, teachers, community). We propose the use of the CEA standards as a guide for any \\ evaluative effort in foreign language programs while designing context-specific tools to address \\ the needs of each program. Finally, we aim to promote the development and dissemination of \\ case studies, resources, and/or journals to enhance the status of language program evaluation \\ within the field of applied linguistics.
}

\section{Keywords}

Program evaluation; language teaching; educational standards; educational quality.

Recibido el 19/09/2019

Aceptado el 10/01/2020 


\section{Introducción}

En los últimos años se ha experimentado un proceso de reconfiguración del sector de organizaciones de enseñanza de lenguas — tanto privadas como públicas - en el que se ha pasado del vínculo tradicional entre profesor y estudiante a otro más enfocado en la relación entre proveedor de servicios y consumidor (White, Hockley, van der Horst y Laughner, 2008). Además, la necesidad de competir en un mercado cada vez más amplio y de mayor calidad, así como la obligación ética y moral de crear programas educativos con valor, conlleva un cambio de paradigma en lo que respecta a la concepción, desarrollo y gestión de organizaciones de enseñanza de lenguas. Esto significa que, desde una perspectiva profesional, el/la director(a) o profesor(a) en programas de lengua ha pasado de ser, en algunos casos, una figura centrada exclusivamente en asuntos académicos — currículum, evaluación de logros individuales, enseñanza, etca añadir a sus funciones la gestión, el liderazgo, la mejora y la coordinación de una organización educativa-preparar presupuestos, cumplir con objetivos, garantizar educación de calidad, búsqueda y administración de recursos, y gestión de empleados, entre otras cosas. Todos estos esfuerzos tienen como foco primordial la creación de un programa que satisfaga las necesidades de sus estudiantes para así garantizar el éxito y el valor del programa.

En lo que respecta al ámbito académico, un creciente número de publicaciones dentro del campo de evaluación y dirección de programas de lengua abogan por la inclusión de procesos evaluativos continuos como factor indispensable en el éxito y valor de programas de lengua (Ecke y Ganz, 2014; Norris, 2016; Pfeiffer y Byrnes, 2009; Watanabe, Norris y González-Lloret, 2009, entre otros). Ya sean evaluaciones internas para mejorar el programa o exigencias externas para justificar cualquier tipo de financiación, estas prácticas evaluativas permiten a los educadores y administradores de programas interpretar, mejorar y garantizar la calidad, el valor y la efectividad del programa, así como los resultados del aprendizaje. A pesar del potencial de este campo, la evaluación de programas ha sido tradicionalmente ignorada por el campo de lingüística aplicada (Norris, 2016). Por ejemplo, la Association Internationale de Linguistique Apliquée (AILA) realizó recientemente un sondeo a nivel nacional en los Estados Unidos para analizar el estado de la educación posgraduada en programas de lingüística aplicada. Los resultados ponen en evidencia el estatus actual de la evaluación de programas de lengua como un componente prescindible en la preparación de sus 
estudiantes (Malone y Donovan, 2014 citado en Norris, 2016). Estos datos pueden parecer alarmantes, especialmente si consideramos que las labores de directores de programas de lenguas pueden no solo centrarse en la gestión de los aspectos académicos de un programa, sino también en dirigir los esfuerzos relacionados con el manejo del programa como organización.

El propósito del presente artículo es doble. Primero, proponer un enfoque de evaluación basado en estándares como punto de partida para garantizar la calidad, efectividad y valor de los programas. Segundo, fomentar el desarrollo de prácticas evaluativas y estudios de caso en programas de enseñanza de lenguas, así como de soportes (revistas, libros, manuales) para la diseminación de buenas prácticas en español con el fin de que los profesionales en el mundo de la enseñanza de lenguas puedan disponer de esta información y usarla para el beneficio de sus propios programas.

A continuación, exponemos una introducción a lo que se interpreta en este artículo por evaluación seguida de una concisa visión histórica del tema y una discusión sobre el potencial que los procesos evaluativos en general, y la evaluación basada en estándares en particular, ofrecen para los programas de lengua. Finalmente, concluimos con la descripción de áreas que sería importante evaluar en un programa de lengua y explicaremos por qué el uso de los estándares de CEA puede ayudar a estos programas a garantizar/demostrar su valor.

\section{2. ¿Qué significa evaluación?}

El término evaluación en español es familiar para la mayoría de profesionales y expertos dentro del campo de la enseñanza de lenguas. Este concepto puede, sin embargo, no encarnar el mismo significado para todos, pues existen dos ideas bien diferenciadas que se identifican con la misma palabra. En primer lugar, existe la interpretación de evaluación como recolección de información y datos para estimar el aprendizaje, conocimiento o habilidades a nivel individual. Este tipo de evaluación se mide con respecto a objetivos educativos o competencias concretas que regulan un curso o un programa específico. En segundo lugar, la evaluación también puede contemplarse desde una perspectiva macro/estructural que se centra en el programa en sí. En este contexto, Norris (2006) describe evaluación de la siguiente manera: 
Recolección de información sobre cualquiera de los elementos que componen un programa educativo con diversos propósitos que tratan primordialmente de comprender, demostrar, mejorar y juzgar el valor de un programa. Este tipo de evaluación presenta evidencias a tener en cuenta sobre los problemas a nivel de programa sin restringirse a ningún tipo de metodología en particular (Norris, 2006: 579)ํ․

Por lo tanto, la diferencia entre ambas nociones de evaluación se puede encontrar tanto en su alcance como en su propósito y metodología (Norris, 2016; Lynch, 1996). Mientras que la evaluación individual busca posicionar a los alumnos dentro de una escala concreta de medición, la evaluación de programas recaba información que ayuda a los evaluadores a tomar decisiones. Para el propósito de este artículo, nos centramos en la segunda definición de evaluación (evaluación de programas), la cual ha recibido menos atención en el campo de la enseñanza de lenguas.

\section{La evaluación de programas}

Los primeros esfuerzos evaluativos a nivel programático se llevaron a cabo en el área de la educación. En la década de 1840 en EE. UU. se comenzaron a realizar evaluaciones sistemáticas con el objetivo de medir el aprendizaje y la efectividad de la enseñanza a través de exámenes orales a escolares. Fue más adelante, en los años 1930, cuando la evaluación comenzó a ser considerada como un movimiento reconocido al establecerse la evaluación basada en objetivos educativos (Stufflebeam y Shinkfield, 2007). Desde entonces, el campo de la evaluación educativa ha ido evolucionando, dando lugar a diferentes enfoques y modelos, los cuales han sido aplicados también a programas de lengua. En los años 60 y 70, las primeras evaluaciones de programas de lengua estaban principalmente orientadas al producto y se centraron en recolectar información sumativa, la cual se utilizaba para determinar el éxito de un programa. De

1 «Evaluation is the gathering of information about any of the variety of elements that constitute educational programs, for a variety of purposes that primarily include understanding, demonstrating, improving, and judging program value. Evaluation brings evidence to bear on the problems of programs, but the nature of that evidence is not restricted to one particular methodology». 
hecho, las primeras evaluaciones de programa dentro del campo de la lingüística aplicada estaban diseñadas para comparar la efectividad de diferentes metodologías y materiales de enseñanza (Beretta, 1992). Fue en los años 80 y 90 cuando las perspectivas sobre la evaluación de programas fueron redirigiéndose hacia la importancia de mirar más allá de los resultados o productos por medio de datos cuantitativos (enfoque positivista), defendiendo la recolección de datos cualitativos (enfoque naturalista) a través de métodos como las entrevistas o las observaciones de clases (Beretta y Davies, 1985; Brown, 1989, 1995; Jacobson, 1982; Long, 1984; Lynch, 1990, 1992). Esta visión desembocó, a finales de los años 90, en el desarrollo de un movimiento conceptual centrado en el uso y el pragmatismo de la evaluación de programas (Kiely y Rea-Dickins, 2005; Norris, 2006, 2009, 2016). Esta visión está fuertemente influenciada por el Enfoque Centrado en el Uso desarrollado por Patton (1997, 2005), el cual causó un gran impacto en las prácticas evaluadoras. Dentro de esta tradición pragmática se promueve la combinación de datos cualitativos y cuantitativos (Lynch, 1996) y la triangulación -el uso de diferentes métodos de recolección de datos que llevan a las mismas conclusiones - para validar los resultados (Allen, 2004).

Algunos autores apuntan a esta visión pragmática de la evaluación como responsable de la falta de propuestas e investigaciones teóricas en materia de evaluación de programas. Stufflebeam y Shinkfield (2007) argumentan que, aunque los expertos en evaluación han sido creativos e influyentes al estimular nuevos enfoques y modelos, estos esfuerzos no han sido acompañados por investigación empírica. Por lo tanto, no existe hasta el momento una evidencia sustancial del funcionamiento de diferentes enfoques evaluativos ni una teoría unificadora sobre la evaluación de programas (Stufflebeam y Shinkfield, 2007). Aun así, los esfuerzos evaluativos a nivel programático en la enseñanza de lenguas han dado lugar a la creación de conceptos, estándares, principios y guías que son valiosos para alcanzar la confiabilidad de un programa. Además, estos han probado ser útiles para revelar líneas de actuación y desarrollo y, así, aumentar el valor de los programas de lengua (Norris et al., 2009).

La evaluación de programas es un campo en expansión que ha motivado un gran número de estudios y modelos orientados a la mejora de programas de lengua. A pesar de su utilidad y su evolución pragmática especializada en programas de lenguas, el campo de evaluación de programas no ha conseguido establecerse (Watanabe et al, 2009) ni encontrar su lugar dentro del campo de adquisición de segundas lenguas, lingüística aplicada o enseñanza de idiomas. No obstante, los trabajos realizados hasta 
la fecha han sentado unos cimientos sólidos desde los que partir para llevar a cabo cualquier esfuerzo evaluador dentro de programas de lengua. A continuación, exponemos las motivaciones y los objetivos en la evaluación de programas.

\section{4. ¿Por qué evaluar programas de lengua?}

Desde su concepción, la noción de evaluación ha sido interpretada como sinónimo de mejoría y desarrollo (Kiely, 2006). La evaluación puede suponer un beneficio para los programas por dos razones. Primero, porque puede aportar evidencia de la calidad del programa; y segundo, porque facilita la mejora y el desarrollo del programa (Jacobson, 1982; Kiely, 2006). Allen (2004) afirma que los encargados potenciales de llevar a cabo estos esfuerzos evaluativos, los educadores, pueden usar la información obtenida de la evaluación para hacer cambios que estén fundamentados en evidencia (cambios curriculares, metodológicos y a nivel administrativo o de programa). También, la evaluación puede servirles para demostrar el impacto de innovaciones educativas a cualquier nivel, el cumplimiento de los objetivos por parte de los estudiantes, y el desarrollo y/o modificación de estos objetivos.

Sin embargo, hay educadores que se han resistido a examinar y reconsiderar sus prácticas en lo que concierne a objetivos educativos, su cumplimiento y lo que esto significa para el programa educativo en general (Sullivan, 2006). Con todo, los órganos de administración superiores u organizaciones de acreditación pueden solicitar del programa de lengua algún tipo de evidencia de su buen funcionamiento, insistiendo así en que se lleven a cabo esfuerzos evaluativos que aporten pruebas de la excelencia del programa. Por lo tanto, podemos referirnos a dos motivaciones (diferentes en su planteamiento) para evaluar un programa. Los programas pueden estar presionados a evaluar sus prácticas por causas externas y/o por causas internas. Así, las evaluaciones pueden estar motivadas por sistemas de acreditación, por la imposición administrativa de demostrar la excelencia del programa o por la ética profesional de los educadores. Watanabe et al. (2009) llevaron a cabo un sondeo en programas de lengua extranjera en EE. UU. sobre las motivaciones para evaluar sus programas. Los resultados muestran que la mayoría de los esfuerzos evaluativos llevados a cabo están motivados por razones externas al programa. Esto puede poner en peligro la integridad y la utilidad de las prácticas evaluativas ya que pueden ser dirigidas hacia un objetivo erróneo y a desarrollar preguntas y herramientas evaluativas que satisfagan los objetivos externos, 
pero no la mejora del programa. Por ejemplo, Davis (2015) encuentra que la evaluación dirigida a satisfacer estas demandas institucionales externas al programa de lengua parece no causar efectos significativos en los procesos educativos. Esto significa que los efectos beneficiosos que una actividad evaluativa puede tener en un programa pueden estar en riesgo si su objetivo es tan solo satisfacer demandas externas. Por lo tanto, Davis (2015) sostiene que la responsabilidad última de que un proceso evaluativo tenga utilidad para el programa recae en los educadores/evaluadores y no en las prácticas externas impuestas, abogando así por una 'cultura de la evaluación' que valore y busque la educación de calidad.

Una de las principales características que resultan ventajosas respecto a los protocolos de evaluación es la posibilidad de adaptar y diseñar las herramientas que mejor puedan analizar el objeto de la evaluación (Lynch, 2003). Las características idiosincráticas y distintivas de cada programa hacen que sea desaconsejable el uso de un método uniforme. Aunque el propósito de la evaluación sea común entre programas de lengua -i.e. buscar la excelencia académica-, diferencias en estructura, objetivos y audiencia hacen que cada esfuerzo evaluativo necesite ser diseñado a medida para cada programa. Por este motivo, es importante resaltar el papel decisivo de los análisis de necesidades para diagnosticar qué áreas necesitan ser optimizadas o para proveer evidencia sobre su buen funcionamiento.

Otro punto a favor de la evaluación de programas de lengua tiene que ver con el actual declive de alumnos inscritos en cursos de lengua en ciertos contextos educativos. Por ejemplo, en Estados Unidos, el informe más reciente de la Modern Language Association (MLA) muestra que el número de estudiantes matriculados en los programas de lenguas extranjeras está disminuyendo. Entre el otoño de 2013 y otoño de 2016, las inscripciones en cursos de idiomas extranjeros en Estados Unidos (a excepción del inglés) cayeron un 9.2\%. Aunque la causa de este declive no está del todo clara $-i$ es consecuencia de una tendencia institucional para reducir la obligatoriedad de cursos de lengua o de un menor interés por parte de los estudiantes o una combinación de ambos? - lo que sí es cierto es que el refinamiento de un programa a través de la evaluación puede reducir al mínimo la probabilidad de que los alumnos abandonen sus estudios de lengua extranjera por motivos relacionados con el valor del programa o sus cursos. Además, todo tipo de programas pueden beneficiarse de procesos de evaluación ya que pueden llegar a contar con una base estructural sólida sobre la que poder implementar estrategias de marketing o reclutamiento basadas en 
satisfacción del alumnado y éxito académico y/o profesional. Adicionalmente, un programa de calidad que es valorado por sus estudiantes favorece la publicidad boca a boca.

\section{Evaluación basada en estándares}

El cumplimento de estándares es, actualmente, la manera más común de definir la calidad educativa tanto en la evaluación como en la acreditación de programas. Por un lado, los gobiernos u órganos acreditadores usan estándares como requisito para la acreditación. Por otro lado, las instituciones educativas usan la evaluación basada en estos estándares para demostrar la calidad de sus programas y para mantenerse acreditados (Hämäläinen, 2003; Shawer, 2013). Los procesos de acreditación, por lo tanto, implican esfuerzos tanto internos como externos al programa para asegurar que las instituciones realizan revisiones para mantener y/o mejorar su calidad a través del cumplimiento de ciertos estándares de calidad. Sin embargo, a pesar de lo que se pueda pensar y del trabajo que conlleva desarrollar una serie de estándares que puedan certificar excelencia académica dentro de un campo concreto, las descripciones de los estándares no son por definición perfectas y tan solo proveen estimaciones de los criterios reales (Stake, 2011). Es decir, que las necesidades y los objetivos de cada programa han de tenerse en cuenta a la hora de cumplir los estándares. Así, este tipo de evaluación no puede conceptualizarse como el seguimiento de una lista de requisitos, sino como una guía que nos lleva a crear herramientas evaluativas idóneas para explorar aspectos concretos de un programa con la mayor precisión posible dentro del marco de cada programa. La evaluación de programas de lengua ha dado lugar, desde una perspectiva profesional, a la creación de estándares para guiar los esfuerzos evaluadores con o sin fines acreditativos. A continuación, resumimos brevemente el propósito de distintos estándares creados por diferentes organizaciones relacionadas con la enseñanza de idiomas.

En el caso de España, el Instituto Cervantes lleva a cabo un sistema de acreditación internacional para centros que imparten español para extranjeros a partir del cumplimiento de un número de estándares o requisitos mínimos. Esta certificación, sin embargo, está diseñada con un propósito diferente al del resto de certificaciones mencionadas a continuación. Tiene como fin hacer llegar la presencia del Instituto Cervantes allá donde no existen centros oficiales. De este modo, estos estándares no están diseñados para guiar una evaluación de un programa de lenguas en el que se tenga 
en cuenta la idiosincrasia del centro. Los requisitos de esta certificación tienen como propósito medir la capacidad de los centros para transmitir los valores y la misión del órgano acreditador. Por lo tanto, debido a que su propósito no es el de guiar la evaluación de la calidad de un programa, esta acreditación no es útil para mejorar programas de lengua.

En el campo de las lenguas extranjeras en EE. UU., el American Council on the Teaching of Foreign Languages (ACTFL) ha publicado estándares para el currículum de lenguas extranjeras (National Standards Collaborative Board, 2015). Estos estándares, tal y como indica el consejo de ACTFL, resultan muy útiles a nivel curricular, pero no tienen como objetivo primordial la excelencia a nivel programático. Por ejemplo, los estándares globales para el aprendizaje de lenguas se basan en cinco principios/áreas fundamentales: la Comunicación, las Culturas, las Conexiones, las Comparaciones y las Comunidades. Estas áreas están entrelazadas y tienen un papel esencial en la formación de los aprendices de lenguas extranjeras. Por tanto, estos estándares son muy valiosos a la hora de construir y evaluar un currículum, pero aún es necesario disponer de otras directrices o estándares que guíen el buen funcionamiento de un programa, más allá de lo estrictamente curricular.

También en EE. UU., Quality Matters ha desarrollado unos estándares de calidad educativa (Quality Matters Standards) que se centran principalmente en medir la calidad de los cursos en línea a nivel de diseño curricular. Los estándares tienen que ver con el resumen del curso e introducción al curso en línea, los objetivos del aprendizaje (o competencias), la evaluación y la medición de los logros individuales, los materiales, las actividades del curso e interacción entre los estudiantes, la tecnología, el apoyo o la ayuda al estudiante y, por último, la accesibilidad y facilidad de uso. De nuevo, estas directrices parecen ser muy provechosas en la actualidad para avalar la calidad del diseño de los cursos en línea - sean de lenguas extranjeras o no-, ya que esta organización (Quality Matters) certifica programas en línea, provocando también una proliferación de talleres y cursos para ayudar a los profesionales a diseñar y construir mejores cursos en línea. Aunque estos estándares son útiles para llevar a cabo buenas prácticas a nivel del diseño de cursos en línea a nivel individual, no son aptos para evaluar la articulación, la progresión y el engranaje de todos los cursos de un programa ni tampoco se aseguran de que el currículum se ajuste a las verdaderas necesidades de los estudiantes. 
Por último, tal y como ocurre con muchos otros aspectos de la enseñanza de lenguas, la evaluación de programas desde una perspectiva profesional se ha desarrollado con mayor fuerza en el campo de la enseñanza de inglés. Un buen ejemplo, es The Commission on English Language Program Accreditation (CEA), una agencia acreditadora de programas de enseñanza de inglés a nivel postsecundario. Esta agencia está formada por profesionales de la enseñanza del inglés y su misión es promover la excelencia en la administración y enseñanza de centros de inglés como segunda lengua o lengua extranjera. Para lograr su objetivo, han establecido una serie de estándares a nivel de programa que han de ser cumplidos como condición para obtener su acreditación (CEA, 2018). Estos estándares buscan garantizar que los programas de inglés provean educación de calidad y son diseñados y revisados por miembros que ejercen su profesión en el campo de la enseñanza de inglés. Sus puntos de actuación son once: misión del programa, currículum, profesorado, instalaciones, capacidad fiscal y administrativa, servicios al estudiante, reclutamiento, duración y estructura del programa, resultados de los estudiantes, quejas de los estudiantes $\mathrm{y}$, por último, desarrollo, planificación y revisión del programa. Estos estándares ayudan a evaluar programas de lenguas a través de procesos de (1) autoevaluación regular y (2) evaluaciones externas por otros profesionales acreditados.

Una vez discutidos los diferentes sistemas de estándares creados por diferentes organizaciones, los creados por la agencia CEA son los más completos y minuciosos, ya que comprenden todos los componentes que constituyen los programas de lenguas. Aunque muchos programas de inglés de EE. UU. solicitan ser acreditados por esta agencia para verificar su excelencia académica, no existen publicaciones académicas acerca de los procesos evaluativos en instituciones. Aun así, estos estándares se encuentran disponibles en línea (CEA, 2018) e incluso se pueden encontrar ejemplos de evaluaciones exhaustivas hechos con estos estándares en programas de lenguas (Weber State University, 2017; West Virginia University, 2016) que pueden servir como guía para cualquier evaluador interesado. En el próximo apartado, se explican los resultados de un análisis que resulta pertinente para corroborar la importancia, la utilidad, las posibilidades, y la aplicabilidad de estos estándares a programas de lengua extranjeras y no solo a programas de inglés. 


\section{6. Áreas de intervención}

En un proyecto relativamente reciente, Watanabe et al. (2009) llevaron a cabo un análisis de las necesidades de diferentes programas universitarios de lengua extranjera como punto de partida para desarrollar prácticas útiles de evaluación de programas. Para este análisis, contaron con 21 participantes de diferentes programas universitarios y centros de lengua, incluyendo jefes de departamento y coordinadores. Además, se usaron cuatro métodos para la recogida de datos: grupos de debate, encuestas breves, entrevistas estructuradas, y sondeos a nivel nacional. Los autores se centraron en diferentes cuestiones relacionadas con la evaluación de programas: (a) los motivos y las presiones para llevar a cabo la evaluación de programas universitarios estadounidenses; (b) los usos esenciales de la evaluación y los métodos de evaluación de programas; (c) las capacidades actuales de los programas de lengua extranjera para desarrollar y usar modelos de evaluación, instrumentos y procedimientos; y (d) las necesidades de desarrollo y las preocupaciones en cuanto a la evaluación en los distintos tipos de programas. De acuerdo con los resultados de este proyecto, los autores hallaron que los componentes principales que están —o deberían estarsujetos a prácticas evaluativas regulares son: (a) la conexión del programa con la institución y la comunidad; (b) la misión del programa; (c) el progreso y los resultados del aprendizaje de los estudiantes; (d) la articulación curricular entre los cursos; (e) las programaciones de los cursos, materiales y libros de texto; (f) la enseñanza, administración, y otros aspectos del desarrollo del trabajo; y (g) las necesidades de los estudiantes y los antecedentes de aprendizaje del idioma de los estudiantes (Watanabe et al., 2009).

Resulta interesante observar la correspondencia entre los resultados relacionados con los componentes sujetos a evaluación en programas de lengua mencionados en el párrafo anterior y los estándares de CEA. Desde un punto de vista práctico, este hallazgo puede resultar muy ventajoso y útil para programas de lengua extranjera, puesto que se han podido identificar los puntos clave de programas de lengua que siempre deben ser susceptibles de una constante valoración o evaluación. Por este motivo, al no existir estándares específicos que se ajusten a las necesidades de varios tipos de programas de lengua extranjera, los estándares de CEA pueden servir como punto de partida para guiar las prácticas evaluativas de todos los componentes de un programa, tales como la misión del programa, el currículum, el profesorado, los resultados del aprendizaje de 
los estudiantes, el desarrollo, la planificación y la revisión del programa, etc. Por supuesto, un estudio o análisis en profundidad de cada componente del programa es necesario para garantizar la calidad del programa, pero los estándares de CEA suponen un punto de arranque para aquellos programas que quieran detectar dificultades en cualquiera de sus componentes con el objetivo de mantener un estatus de calidad educativa.

\subsection{El currículum}

El currículum es una de las partes fundamentales de un programa de lengua extranjera. El diseño curricular describe los contenidos a la vez que guía su impartición y secuenciación. Se asume que la estructura curricular debe reflejar las necesidades de los aprendices (Richards, 2001). Estas necesidades han de estar basadas en resultados objetivos y no en pensamiento intuitivo (Stake, 2011). De esta manera se pueden controlar la subjetividad y el sesgo personal de los evaluadores. El procedimiento por el cual se recaba esta información es conocido como análisis de necesidades. Los resultados de este análisis permiten a los educadores adaptar sus programas a las necesidades reales de sus estudiantes, así como desvelar el buen o mal funcionamiento de los componentes de su programa. De esta forma, Richards (2001) apunta que los análisis de necesidades pueden ser usados para diferentes propósitos:

- para descubrir qué habilidades lingüísticas necesita un estudiante para llevar a cabo una actividad específica (ser guía turístico, ser mánager, ser estudiante universitario, aprobar un examen de proficiencia, etc);

- para ayudar a determinar si un curso existente refleja las necesidades de estudiantes potenciales;

- para determinar qué estudiantes dentro de un grupo necesitan más formación en habilidades lingüísticas específicas;

- para identificar un cambio de dirección del programa;

- para identificar una brecha entre lo que los estudiantes pueden hacer y lo que necesitan poder hacer;

- para recolectar información sobre un problema específico que estén experimentando los estudiantes.

Por lo tanto, el análisis de necesidades forma parte tanto de la concepción de un programa de lengua como de su revisión y refinamiento. Los estándares de CEA (2018) relacionados con el currículum hacen hincapié en la estrecha relación que ha de existir 
entre el currículum y las necesidades de los estudiantes, así como los objetivos y la misión de la institución donde se enmarca: «Estándar de currículum 1: El currículum es consistente con la misión del programa o institución, apropiado para lograr los objetivos de la organización y abordar las necesidades de los estudiantes, y está disponible por escrito» ${ }^{2}$ (CEA, 2018, p. 9). Así, cualquier educador o administrador que decida aplicar este estándar para evaluar su currículum, necesitará crear una serie de herramientas que le ayuden a recabar la información necesaria para construir un currículum que refleje las necesidades de sus estudiantes o para probar la estrecha relación que existe entre las necesidades de sus estudiantes y el diseño de su currículum. Para probar cualquiera de estas dos posibilidades, se podrían desarrollar instrumentos que respondan a diferentes preguntas, por ejemplo:

- ¿Por qué y para qué asisten los estudiantes a este centro de lengua?

- ¿Qué quieren obtener del programa?

- ¿Cuál es el nivel de competencia lingüística general de entrada al programa?

- ¿Se corresponden los objetivos curriculares con los resultados del aprendizaje?

- ¿Qué les ha sido útil o no útil a los estudiantes que han terminado el programa?

- ¿Consiguen sus metas lingüísticas los estudiantes que terminan el programa?

Dependiendo de la naturaleza de cada programa, las preguntas serán diferentes y estarán adaptadas al contexto de la institución. A partir de los datos obtenidos en el análisis de necesidades, los educadores podrán tomar decisiones curriculares bien fundamentadas en datos objetivos que proveerán oportunidades para crear programas valiosos para sus participantes.

\footnotetext{
${ }^{2}$ «Curriculum Standard 1: The curriculum is consistent with the mission of the program or language institution, appropriate to achieve the organization's goals and meet assessed student needs, and available in writing».
} 


\subsection{Más allá del currículum}

Los programas de lengua poseen más componentes que pueden ayudar a mejorar la calidad de un programa y, por lo tanto, pueden ser evaluados. Por ejemplo, el método de enseñanza, la misión de un programa, el profesorado, las instalaciones, los equipos y procesos administrativos, los servicios al estudiante, publicidad/marketing, los resultados del aprendizaje, quejas de los estudiantes, etc. Aunque la evaluación de todos estos componentes puede no ser aplicable a ciertas instituciones, los estándares de CEA recogen estos aspectos programáticos y pueden facilitar su evaluación. Por ejemplo, algunos programas de lengua están enmarcados en organismos de educación superior que poseen servicios al estudiante, instalaciones y equipos administrativos a nivel institucional fuera de la responsabilidad de los administradores del programa de lengua. Otros componentes como los resultados del aprendizaje sí son relevantes para los programas de lengua en general. La evaluación de resultados del aprendizaje ha motivado varios estudios que ejemplifican cómo medir el aprendizaje de los objetivos curriculares por parte de los estudiantes para mejorar la estructura y la efectividad de un programa (véase Norris y Davis, 2015). Por su parte, CEA aborda este tema con el siguiente estándar: «Estándar de resultados del aprendizaje 2: El programa o institución documenta por escrito si los estudiantes están preparados para pasar al siguiente nivel o para salir del programa de estudio usando instrumentos que evalúan el aprendizaje de los estudiantes en cursos tomados dentro del currículum» ${ }^{3}$ (CEA, 2018, p. 39). Con respecto a la evaluación de este estándar, se pueden examinar, por ejemplo, los instrumentos utilizados para medir el progreso de los estudiantes, la alineación de estos instrumentos con el currículum y los objetivos, la efectividad de estos instrumentos para evaluar los resultados del aprendizaje o hacer un seguimiento de los datos con relación a los resultados del aprendizaje por parte de los estudiantes. Toda la información recabada en este tema puede ayudar a refinar los métodos de medición de resultados a la vez que se pone de manifiesto cuánto aprenden los estudiantes en un programa.

\footnotetext{
3 «Student Achievement Standard 2: The program or language institution documents in writing whether students are ready to progress to the next level or to exit the program of study, using instruments or procedures that appropriately assess the achievement of student learning outcomes for courses taken within the curriculum».
} 


\section{Conclusiones y líneas de actuación}

El área de evaluación de programas de lengua es relativamente novedosa y, por este motivo, no existen muchas publicaciones de investigaciones que presenten resultados con datos concluyentes. No obstante, la mayor parte de estas publicaciones son de gran utilidad, ya que aportan directrices y pautas que pueden guiar a evaluadores para identificar posibles áreas específicas dentro de su programa que requieran atención, para mantener un buen funcionamiento de todos los componentes que constituyen el programa o para obtener mejores prácticas que permitan alcanzar la excelencia académica. También recomendamos para este propósito el uso de los estándares de CEA (2018) debido a su utilidad a la hora de guiar esfuerzos evaluativos en programas de lengua. A falta de un set de estándares expuestos por un órgano acreditador específico para programas de lengua extranjera en general, estos estándares proveen una visión general de qué componentes han de ser examinados en una evaluación de programa.

Existe cierta inquietud en la actualidad por la innovación docente o educativa y con el uso de nuevas herramientas y estrategias que fomentan el aprendizaje. El propósito de este artículo no es en absoluto rechazar la innovación educativa, sino más bien poner de manifiesto que antes de invertir en innovación, los programas de lengua deberían focalizar sus esfuerzos en analizar cuáles son las necesidades reales de las partes involucradas (estudiantes, profesores) en estos programas. Una incorporación innovadora debe tener como objetivo solucionar un problema documentado por datos objetivos obtenidos con cualquier herramienta evaluadora. Esta herramienta, a su vez, nos permitirá observar si la implementación de dicha innovación ha servido para mejorar el programa. Como se mencionó anteriormente, un análisis de necesidades permite tener una visión global de cuáles son las mejores prácticas del programa, sus fortalezas y aquellas áreas que requieren atención para mejorar y poder lograr la excelencia en el ámbito académico. Su uso es recomendado como base para cualquier cambio en el programa. Del mismo modo, se debe tener en cuenta que llevar a cabo estos análisis o evaluaciones a nivel programático no consiste solo en centrar la mirada en el currículum del programa. Si bien ésta es una parte primordial de los programas de lengua, no se trata del único aspecto a evaluar, ya que existen muchos ámbitos dentro de un programa de lengua que van más allá del diseño curricular tales como la misión 
del programa, el profesorado, los resultados del aprendizaje de los estudiantes, e incluso el mismo proceso de planificación y evaluación del programa.

Aunque parezca obvio, no debemos olvidar que los objetivos de los cursos de un programa de lengua deben estar directamente relacionados con el aprendizaje de la lengua y la mejora de las habilidades lingüísticas por parte de los estudiantes. Es ventajoso contar con estándares relacionados con el diseño o la forma de los cursos, pero estos deben ser siempre complementados con estándares que permitan evaluar la calidad del contenido curricular y la correspondencia entre los objetivos con los resultados reales del aprendizaje en relación a las distintas habilidades lingüísticas.

Otro aspecto fundamental que debemos tener presente es que llevar a cabo el proceso evaluativo de un programa no puede recaer exclusivamente en la directora o el director de un programa. Si bien, el/la director(a) juega un papel importante en este procedimiento, ya que son imprescindibles su experiencia y su conocimiento del programa, la evaluación debe ser un esfuerzo común por parte de profesores, coordinadores, y administradores. Es importante resaltar que la profesión de director de un programa difícilmente admite la incorporación de obligaciones evaluadoras. La institución debería reconocer que la evaluación de un programa es un beneficio común y ha de hacer partícipe a la comunidad educativa en general. Por lo tanto, es importante enfatizar que la cuestión de evaluación de programas de lengua es un asunto académico. Tanto los programas de lengua como los campos de la evaluación y la lingüística aplicada se beneficiarían si futuros administradores de programa y profesionales de la enseñanza de lenguas estuvieran académicamente preparados para lo que implica trabajar en un contexto educativo. De esta manera, se ayudaría a la creación de un perfil profesional más completo dirigido a la gestión y administración de organismos educativos. Para predicar con el ejemplo, sería interesante expandir los sondeos sobre el perfil laboral y formativo de los profesionales en el campo de lengua extranjera (ver Muñoz-Basols et al., 2017 para educadores de ELE) para explorar sus responsabilidades y experiencias con respecto a la gestión y evaluación de programas y su preparación académica. Esto nos daría una idea del estado del campo con respecto a la dirección y evaluación de programas de lengua extranjera.

Finalmente, cabe mencionar que están proliferando cada vez más los canales de divulgación de investigación en materia de evaluación de programas de lengua. Hoy en día, no es extraño encontrar presentaciones profesionales y académicas sobre evaluación en conferencias sobre enseñanza de lenguas y actualmente existen varios 
libros sobre este tema (Alderson y Beretta, 1992; Allen, 2004; Davis y McKay, 2018; Davis, Norris, Malone, McKay y Son, 2018; Kiely y Rea-Dickins, 2005; Lynch, 1996, 2003; Norris, Davis, Sinicrope y Watanabe, 2009, entre otros). Sin embargo, el campo se beneficiaría enormemente de la publicación de estudios de caso que ayuden a esbozar una teoría unificadora de evaluación de programas para comprobar empíricamente los efectos de la evaluación en diferentes componentes programáticos. Esto es posible a través del análisis de datos longitudinales que permitan observar los efectos de los ajustes al programa. Quizás sería también beneficioso el desarrollo de materiales y estudios en español para facilitar el acceso a personas de habla hispana que tengan dentro de sus responsabilidades la coordinación o dirección de programas de lengua.

\section{Referencias bibliográficas}

ALDERSON, J. C., \& BERETTA, A. (1992). Evaluating Second Language Education. Cambridge University Press.

ALLEN, M. J. (2004). Assessing academic programs in higher education. Bolton, MA: Anker Publishing Company.

BERETTA, A. (1992). «Evaluation of language education: An overview ». En J. C. ALDERSON \& A. BERETTA (Eds.), Evaluating Second Language Education (pp. 524). Cambridge, UK: Cambridge University Press.

BERETTA, A., \& DAVIES, A. (1985). «Evaluation of the Bangalore Project ». ELT Journal, 39(2), 121-127.

BROWN, J. D. (1989). «Language program evaluation: A synthesis of existing possibilities». En R. JOHNSON (Ed.), The second language curriculum (pp. 222-242). Cambridge, UK: Cambridge University Press.

BROWN, J. D. (1995). «Language Program Evaluation: Decisions, Problems and Solutions». Annual Review of Applied Linguistics, 15, 227-248.

CEA. (2018). «CEA standards for English language programs and institutions». Retrieved from http://cea-accredit.org/images/pdfs/2018_CEA_Standards.pdf

DAVIS, J. M. (2015). «The usefulness of accreditation-mandated outcomes assessment: Trends in university foreign language programs». En J. NORRIS \& J. DAVIS (Eds.), Student learning outcomes assessment in college foreign language programs (pp. 135). Honolulu, HI: University of Hawai 'i, National Foreign Language Resource Center.

DAVIS, J. M., \& MCKAY, T. H. (2018). A Guide to Useful Evaluation of Language Programs. Georgetown University Press.

DAVIS, J. M., \& NORRIS, J. M. (2015). Student Learning Outcomes Assessment in College Foreign Language Programs. National Foreign Language Resource Center.

DAVIS, J. M., NORRIS, J. M., MALONE, M. E., MCKAY, T. H., \& SON, Y.-A. (2018). Useful Assessment and Evaluation in Language Education. Georgetown University Press. 
ECKE, P., \& GANZ, A. (2014). «Student analytics and the longitudinal evaluation of language programs». En J. NORRIS \& N. MILLS (Eds.), Issues in language program direction: Innovation and accountability in language program evaluation (pp. 6282). Boston: Cengage Learning.

HÄMÄLÄINEN, K. (2003). «Common Standards for Programme Evaluations and Accreditation? ». European Journal of Education, 38(3), 291-300.

JACOBSON, P. L. H. (1982). «Using Evaluation to Improve Foreign Language Education». The Modern Language Journal, 66(3), 284-291.

KIELY, R. (2006). «Evaluation, Innovation, and Ownership in Language Programs». The Modern Language Journal, 90(4), 597-601.

KIELY, R., \& REA-DICKINS, P. (2005). Program Evaluation in Language Education. New York: Palgrave Macmillan.

LONG, M. H. (1984). «Process and Product in ESL Program Evaluation». TESOL Quarterly, 18(3), 409-425.

LYNCH, B. K. (1990). "A Context-Adaptive Model for Program Evaluation». TESOL Quarterly, 24(1), 23-42.

LYNCH, B. K. (1992). «Evaluating a program inside and out». En J. ALDERSON \& A. BERETTA (Eds.), Evaluating second language education (pp. 61-99). Cambridge, UK: Cambridge University Press.

LYNCH, B. K. (1996). Language Program Evaluation: Theory and Practice. Cambridge, UK: Cambridge University Press.

MALONE, M., \& DONOVAN, A. (2014). The state of applied linguistics in the United States: results of a nationwide survey. Presentado en the Annual conference of the International Association of Applied Linguistics (AILA), Brisbane, Australia.

MUÑOZ-BASOLS, J., RODRÍGUEZ-LIFANTE, A., \& CRUZ-MOYA, O. (2017). «Perfil laboral, formativo e investigador del profesional de español como lengua extranjera o segunda (ELE/EL2): datos cuantitativos y cualitativos». Journal of Spanish Language Teaching, 4(1), 1-34.

NATIONAL STANDARDS COLLABORATIVE BOARD. (2015). World-readiness standards for learning languages (4th ed.). Author Alexandria, VA.

NORRIS, J. (2006). «The Why (and How) of Assessing Student Learning Outcomes in College Foreign Language Programs». The Modern Language Journal, 90(4), 576583.

NORRIS, J. (2016). «Language Program Evaluation». The Modern Language Journal, 100(S1), 169-189.

NORRIS, J. M. (2009). «Understanding and improving language education through program evaluation: Introduction to the special issue». Language Teaching Research, 13(1), 7-13.

NORRIS, J. M., DAVIS, J. M., SINICROPE, C., \& WATANABE, Y. (2009). Toward Useful Program Evaluation in College Foreign Language Education. Honolulu, HI: University of Hawai'i, National Foreign Language Resource Center.

PATTON, M. Q. (1998). Utilization-focused evaluation: The new century text. Thousand Oaks, CA: Sage. 
PATTON, M. Q. (2005). «Utilization-Focused Evaluation». En S. MATHISON (Ed.), Encyclopedia of evaluation (pp. 429-432). Thousand Oaks, CA: Sage.

PFEIFFER, P. C., \& BYRNES, H. (2009). «Curriculum, learning, and the identity of majors: A case study of program outcomes evaluation». En J. NORRIS, J. DAVIS, C. SINICROPE, \& Y. WATANABE (Eds.), Toward useful program evaluation in college foreign language education (pp. 183-208). Honolulu, HI: National Foreign Language Resource Center Honolulu, HI.

RICHARDS, J. C. (2001). Curriculum development in language teaching. Cambridge University Press

SHAWER, S. F. (2013). «Accreditation and standards-driven program evaluation: implications for program quality assurance and stakeholder professional development». Quality \& Quantity, 47(5), 2883-2913.

STAKE, R. E. (2011). Standards-Based and Responsive Evaluation. Thousand Oaks, CA: SAGE.

STUFFLEBEAM, D. L., \& SHINKFIELD, A. J. (2007). Evaluation Theory, Models, and Applications. San Francisco, CA: Jossey-Bass.

SULLIVAN, J. H. (2006). «The Importance of Program Evaluation in Collegiate Foreign Language Programs». The Modern Language Journal, 90(4), 590-593.

WATANABE, Y., NORRIS, J. M., \& GONZÁLEZ-LLORET, M. (2009). «Identifying and responding to evaluation needs in college foreign language programs». En J. Norris, J. Davis, C. Sinicrope, \& Y. Watanabe (Eds.), Toward useful program evaluation in college foreign language education (pp. 5-56). Honolulu, HI: University of Hawaii, National Foreign Language Resource Center Honolulu, HI.

WEBER STATE UNIVERSITY (2017). Extraído de https://apps.weber.edu/wsuimages/assessment/ProgramReview/LEAP/Self_Stu dy.pdf

WEST VIRGINIA UNIVERSITY (2016). Extraído de https://www.wwu.edu/ee/accreditation/2C17/EE2C17IEPSelfStudy.pdf

WHITE, R., HOCKLEY, A., VAN DER HORST J., J., \& LAUGHNER, M. (2008). From teacher to manager: Managing language teaching organizations. Cambridge, UK: Cambridge University Pres 